\title{
A QUASI-COMMUTATIVE RING THAT IS NOT NEO-COMMUTATIVE
}

\author{
IRVING KAPLANSKY
}

(Communicated by Ken Goodearl)

\begin{abstract}
An example of a ring that is quasi-commutative but not neo-commutative is given.
\end{abstract}

In $[2$, p. 73$]$ I defined a neo-commutative ring to be one where the product of any two finitely generated ideals is finitely generated. (All ideals in this note are two-sided.) In [1] Belluce weakened the condition by assuming that the radical of the product of any two principal ideals is the radical of a finitely generated ideal. (The radical of an ideal is the intersection of the prime ideals containing it.) At the end of his paper he asked whether the two conditions are distinct. The answer: they are.

Over a field $k$ let $F$ be the free algebra with unit generated by a countably infinite set of indeterminates $x_{1}, x_{2}, \ldots$. Let $M$ be the ideal generated by the $x_{i}$ 's. Thus $F=k+M$. Let $R=F / M^{4}$. $R$ has exactly one prime ideal (the image of $M$ ), so it is quasi-commutative by default. I claim that $R$ is not neocommutative. Let $I$ be the ideal in $R$ generated by $x_{1}$ (by abuse of notation I still write $x_{1}$ for the homomorphic image and similarly for other elements). $I^{2}$ is the set of all $k$-linear combinations of $x_{1}^{2}, x_{i} x_{1}^{2}, x_{1} x_{i} x_{1}, x_{1}^{2} x_{i}$ (all longer products are 0$). I^{2}$ is not finitely generated; in an alleged finite generation only a finite number of $x_{1} x_{i} x_{1}$ would occur, leaving us helpless to account for the rest.

\section{REFERENCES}

1. L. P. Belluce, Spectral spaces and non-commutative rings, Comm. Algebra 19 (1991), 1855-1865.

2. I. Kaplansky, Topics in commutative ring theory, Univ. of Chicago Math. Dept. mimeographed notes, 1974.

Mathematical Sciences Research Institute, Berkeley, California 94720

Received by the editors October 25, 1993.

1991 Mathematics Subject Classification. Primary 16A15. 